\title{
Threshold dimorphism in ejaculate characteristics in the squid Loligo bleekeri
}

\author{
Yoko Iwata*, Yasunori Sakurai
}

Graduate School of Fisheries Sciences, Hokkaido University, 3-1-1 Minato-cho, Hakodate, Hokkaido 041-8611, Japan

\begin{abstract}
Male dimorphism has been thought to correlate with alternative reproductive behaviors. Alternative reproductive behaviors promote asymmetry in sperm competition, and the differences in fertilization success could promote adaptations in ejaculate characteristics in relation to each reproductive behavior. Using allometric analysis, we show that ejaculate dimorphism clearly exists in males of the squid Loligo bleekeri, a cephalopod species with body size-related alternative mating behaviors. A morphological switch point was detected for internal characters: larger individuals produced discontinuously longer spermatophores than did smaller individuals, although no switch point was detected for external characteristics (fin length, fin width, head width, mantle width, tentacle length and hectocotylus length) except for bimodal body size. This clear internal switch point could be an adaptation to the characteristic alternative mating behaviors of loliginid squid, in which males use different mating tactics to pass spermatophores to different sperm storage sites in and on the females. Our results indicated that alternative reproductive behaviors can result in morphological adjustment in internal characteristics
\end{abstract}

KEY WORDS: Male dimorphism $\cdot$ Sperm competition $\cdot$ Alternative reproductive behavior $\cdot$ Squid $\cdot$ Loligo bleekeri

Resale or republication not permitted without written consent of the publisher

\section{INTRODUCTION}

In many species the expression of secondary sexual traits of individuals varies within a sex (Andersson 1994). Game theory suggests that each individual will choose a phenotype that achieves the highest fitness depending on its competitive ability or social status (Gross 1996). Such a conditional strategy will have a phenotypic switch point where the fitnesses of the 2 phenotypes are equal (Gross 1996). The most common conditional reproductive strategy has 2 alternative reproductive behaviors that depend on body size ('pair mating' and 'sneaking'), and such alternative reproductive behaviors promote the evolution of male dimorphism (Eberhard 1982, Gross 1996, Taborsky 1998). Males in some species are dimorphic at maturation, with large males having fully developed secondary sexual characters and small males having less developed secondary sexual characters (e.g. Eberhard \& Gutiérrez 1991). Male dimorphism would be favored by heterogeneous selection for discrete alternative mating behaviors. Thus, finding a morphological switch point is im- portant for understanding status-dependent fitness functions of alternative mating behaviors.

Alternative mating behaviors result in not only external male dimorphism but also internal morphological and physiological adaptations. Theoretical models predict that sneaker males with a constantly high sperm competition risk will expend reproductive effort on sperm expenditure to compensate for a disadvantage in behavioral competition (Parker 1990a,b, Parker et al. 1997). Sperm competition risk in other species also affects sperm investment patterns such as ejaculate characters (Snook 2005).

Studies of the ejaculate strategies associated with alternative mating behaviors in animals that fertilize either externally or internally support the predictions of the sperm competition theory (e.g. Gage \& Barnard 1996, Leach \& Montgomerie 2000, Nicholls et al. 2001, Evans et al. 2003). In the animals examined, the ejaculate and sperm storage sites used for each alternative mating behavior were not perfectly separated even though the timing of the sperm release and/or the mating orders differred among males. Female loliginid 
squid, however, have 2 distinct sperm storage sites (the seminal receptacle near the mouth and the opening of the oviduct within the mantle cavity), which corresponds to alternative mating behaviors (Hanlon \& Messenger 1996). Loliginid squids form dense spawning aggregations on coastal spawning grounds, and males pair temporarily with females in order to mate (Hanlon \& Messenger 1996). These spawning aggregations usually tend to have more males than females and the mating behaviors that males use correlate with their body size: large males pair with females and copulate in the male parallel position by attaching spermatophores to the female's oviduct in the mantle cavity, and small sneaker males mate in the head-to-head position by attaching spermatophores near the mouth of females that have already paired with other males (Hanlon et al. 1997, 2002). Sneaker males fertilize fewer eggs than paired males do (Iwata et al. 2005). This suggests that sperm stored near the oviduct have an advantage, perhaps because they are stored closer to the eggs being spawned than are sperm stored in the seminal receptacle near the mouth. These size-dependent alternative mating behaviors and the differences in fertilization success could promote strategic ejaculation in males specializing on different sperm-passing sites. Furthermore, loliginid males pass sperm as spermatophores, enclosing the spermatozoa within a hard shell, and males store several hundred spermatophores when they mature. Therefore, the ejaculate characteristics associated with a male's status can be measured more easily in loliginid squid than in other species that release sperm as milt. Thus, loliginid squid are ideally suited to study how ejaculate characteristics are related to alternative mating behaviors.

In this study, we analyzed the presence of a male morphological switch point for both external and internal characteristics in the squid Loligo bleekeri to determine whether alternative mating behaviors can result in male dimorphism.

\section{MATERIALS AND METHODS}

Sample collection. Samples of Loligo bleekeri were collected in inshore set nets in Aomori during the spawning seasons: February to March in 2004 and January to April in 2005. In 2004, we sampled 154 individuals randomly of which 72 individuals were males (size range, 136 to $341 \mathrm{~mm}$ ). In 2005, we sampled 611 individuals from the commercially fished squid separated among 3 size classes: large $(<18$ ind./ $3 \mathrm{~kg})$, medium (18 to 28 ind. $/ 3 \mathrm{~kg}$ ) and small (>28 ind./ per $3 \mathrm{~kg}$ ). To cover all of the size range of $L$. bleekeri we sampled $12 \mathrm{~kg}$ from the large size class (162 males ranging from 242 to $392 \mathrm{~mm}$ from 165 total individuals ranging from 242 to $392 \mathrm{~mm}$ ),
$8 \mathrm{~kg}$ from the medium size class $(76$ males ranging from 221 to $342 \mathrm{~mm}$ from 171 total individuals ranging from 204 to $342 \mathrm{~mm}$ ) and $8 \mathrm{~kg}$ from the small size class (92 males ranging from 152 to $242 \mathrm{~mm}$ from 275 total individuals ranging from 126 to $252 \mathrm{~mm}$ ). The body size frequency distribution was calculated using the size distribution of the measured samples and catch weight of each size class on each sampling day. Of the 611 individuals measured 330 individuals were males. In both years all individuals were determined to be fully mature according to the maturity stage indices described in Perez et al. (2002) for L. plei.

For each male, we measured the mantle length, fin length, fin width, head width, mantle width, tentacle length and hectocotylus length (4th arm specialized for transferring spermatophores to females). Furthermore, we randomly selected 10 spermatophores from the spermatophore storage organ of each male and measured their lengths using electronic slide calipers under a stereomicroscope. Mean and standard deviation of spermatophore length were calculated for each male. A total of 61 individuals in 2005 had only broken spermatophores in their spermatophore storage organ, so the spermatophore length was measured in the 269 remaining males in 2005, excluding those with damaged spermatophores.

Once spermatophores are passed onto a female's body during copulation, sperm contents are ejaculated from the spermatophore shell. The contents attach to the female body as a 'sperm mass,' in which milt are packed into a thin pouch. For female samples we examined the opening of the oviduct and around the seminal receptacle to determine whether a sperm mass was attached. If a sperm mass was observed in the opening of the oviduct and/or around the seminal receptacle, we noted the shape of the sperm mass.

Data analysis. If more than one mode was observed in body size distribution, a normal mixture model was fitted to the distribution to describe a multi-modality of the body size (Fraley \& Raftery 2002). The body size, $x$, within each size range was assumed to be normally distributed, with a density of:

$$
f_{i}(x ; \mu, \sigma)=\frac{1}{\sqrt{2 \pi \sigma^{2}}} \mathrm{e}^{-(x-\mu)^{2} /\left(2 \sigma^{2}\right)}
$$

where $\mu$ is mean and $\sigma^{2}$ is variance. The overall size distribution is thus a mixture of normal components:

$$
f(x)=\sum_{i=1}^{k} \lambda_{i} f_{i}\left(x_{i} \mu_{i}, \sigma_{i}\right)
$$

with one component for each size mode, the multipliers $\lambda_{i}$ representing the proportion that each size mode contributes to the entire distribution.

For the 72 and 330 males measured in 2004 and 2005, respectively, we tested for male dimorphism 
using the method of Eberhard \& Guitiérrez (1991). First, we fitted the partial regression equation to determine whether the relationship between mantle length and each characteristic was nonlinear, which would indicate a potential dimorphism. The equation is:

$$
\ln Y=\alpha_{0}+\alpha_{1} \ln X+\alpha_{2} \ln X^{2}+\varepsilon
$$

(Model 1)

where $Y$ is the length of each character, $X$ is the mantle length, $\alpha_{i}$ is the regression coefficient and $\varepsilon$ is the random component assuming a normal distribution and homogenous variances. An F-test was conducted for the null hypothesis $H_{0}: \alpha_{2}=0$. If the coefficient $\alpha_{2}$ did not significantly differ from zero, we concluded that the characteristics showed no significant deviation from linearity and that additional analysis was not necessary. If $\alpha_{2}$ differed significantly from zero, we concluded that the relationship was nonlinear, indicating the possible existence of dimorphism.

If the analysis indicated the relationship was nonlinear, a second analysis was conducted to determine if there was a switch point, which was defined as the point where (1) the linear slope of mantle length $(X)$ versus each characteristic $(Y)$ changed, and (2) the change in $Y$ was discontinuous. To test the discontinuity at a switch point we fitted the partial regression equation:

$$
Y=\beta_{0}+\beta_{1} X+\beta_{2}\left(X-X_{0}\right) D+\beta_{3} D+\varepsilon
$$

in which $Y$ is the measurement of each characteristic, $X$ is the mantle length, $X_{0}$ is the proposed switch point, $D$ is the conditional constant $\left(D=0\right.$ when $X<X_{0}$ and $D$ $=1$ when $\left.X_{0}<X\right), \beta_{\mathrm{i}}$ is the regression coefficient and $\varepsilon$ is the random component assuming a normal distribution and homogenous variance. To determine the switch point that gives the highest adjusted $\mathrm{R}^{2}$ value, various $X_{0}$ were substituted in Model 2 with $5 \mathrm{~mm}$ steps through th observed mantle length (155 to $340 \mathrm{~mm}$ ). Using the best-fit switch point, a $t$-test was used to test the null hypothesis $H_{0}: \beta_{3}=0$. If the coefficient $\beta_{3}$ differed significantly from zero, we concluded that dimorphism occurred and it was discontinuous at the switch point.

If coefficient $\beta_{3}$ did not significantly differ from zero, a third analysis was conducted to test the change of liner slope of mantle length and each characteristic at the switch point. We fitted the partial regression equation:

$$
Y=\beta_{0}+\beta_{1} X+\beta_{2}\left(X-X_{0}\right) D+\varepsilon
$$

(Model 3)

in which each term was defined as in Model 2 and the best switch point was determined in the same manner as in Model 2. Using the best switch point in Model 3, a $t$-test was used to test the null hypothesis $H_{0}: \beta_{2}=0$. If coefficient $\beta_{2}$ differed significantly from zero, we concluded that dimorphism occurred with a significant dif- ference in the slopes on either side of the switch point, but it was not discontinuous at the switch point.

\section{RESULTS}

In 2004, male mantle lengths ranged from 136 to $341 \mathrm{~mm}(\mathrm{n}=72)$ and varied more than those of females (164 to $258 \mathrm{~mm}, \mathrm{n}=82$, Fig. 1A). The body size distribution of males and females was unimodal, but male samples contained more individuals of small size (Fig. 1A). In 2005, male mantle lengths ranged from 152 to $392 \mathrm{~mm}(\mathrm{n}=330)$ and varied more than those of females (126 to $280 \mathrm{~mm}, \mathrm{n}=281$, Fig. 1B). The body size distribution of males was bimodal with 2 modes at mantle lengths 175 to $200 \mathrm{~mm}$ and 275 to $300 \mathrm{~mm}$, and the distribution of females was unimodal (Fig. 1B). We fitted a normal mixture model to the size distribution of males. The best fitting model was the mixture of 2 normal distributions, which included a small $(\lambda=0.204, \mu=$ 173.2, $\sigma=18.20)$ and a large one $(\lambda=0.796, \mu=270.6$, $\sigma=29.57$ ). The cross point of the 2 normal distributions was located at mantle length $205 \mathrm{~mm}$.

Table 1 shows the results of analyses for dimorphism in the 7 characteristics examined in both years. In 2004 hectocotylus length and spermatophore length had significant values of $\alpha_{2}$ in Model 1. With Model 2, spermatophore length showed significant discontinuities
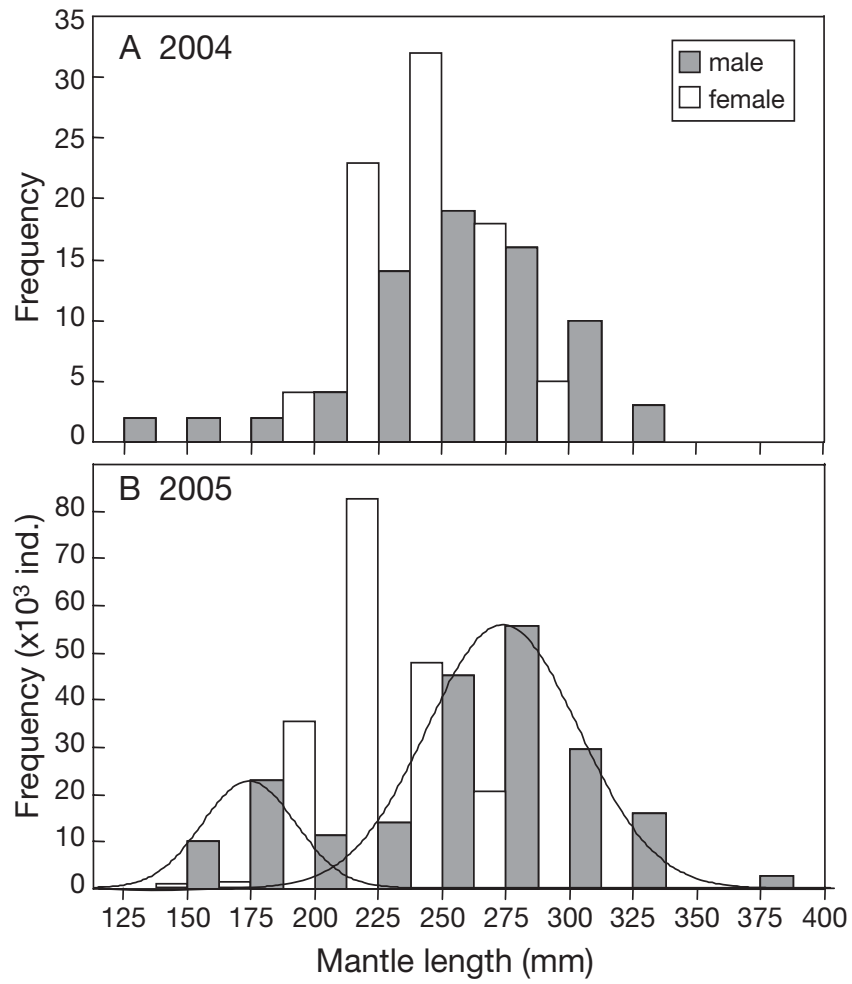

Fig. 1. Loligo bleekeri. Frequency distribution of mature body size in (A) 2004 and (B) 2005 
(reject $\beta_{3}=0$ ), which gave statistical evidence of threshold dimorphism (Fig. 2A). Hectocotylus length was analyzed with Model 3, and the coefficient $\beta_{2}$ did not differ significantly from zero $(t$-test, coefficient $=0.12, t=1.26, \mathrm{p}=0.21$ ), which indicated that there was no dimorphism. In 2005, fin width and spermatophore length had significant values of $\alpha_{2}$ in Model 1. With Model 2, spermatophore length showed significant discontinuities (reject $\beta_{3}=0$ ), which gave statistical evidence of threshold dimorphism (Fig. 2B). Fin width was analyzed with Model 3 and the coefficient $\beta_{2}$ did not significantly differ from zero $(t$-test, coefficient $=-0.02, t=-1.85$, $\mathrm{p}=0.07$ ), which indicated that there was no dimorphism. Thus, none of the external body characteristics (fin length, fin width, head width, mantle width, tentacle length or hectocotylus length) showed a significant deviation from linearity, and spermatophore length showed dimorphism with a discontinuous switch point in both years. The best-fit switch point for spermatophore length based on Model 2 occurred at a mantle length $207 \mathrm{~mm}$ in 2004 and at $221 \mathrm{~mm}$ in 2005; males smaller than this size had discontinuously shorter spermatophores than did larger males (the estimated differences in spermatophore length between the longest short type and the shortest long type were $3.33 \mathrm{~mm}$ in 2004 and $3.22 \mathrm{~mm}$ in 2005, respectively). In contrast, there was little variation in spermatophore length within each individual (average SD of 10 spermatophore lengths $=0.439$ in 2004 and 0.387 in 2005). Spermatophore lengths were positively related to mantle length both in the short type and in the long type in both years (in 2004, long type: $\mathrm{n}=65, Y=$ $0.022 X+9.755, \mathrm{R}^{2}=0.480, F_{1,63}=58.15, \mathrm{p}<0.001$, short type: $\mathrm{n}=7, Y=0.029 X+4.947, \mathrm{R}^{2}=0.838, F_{1,5}=25.91$, $\mathrm{p}<0.01$, Fig. $2 \mathrm{~A}_{i}$ in 2005, long type: $\mathrm{n}=212, Y=$ $0.021 X+9.703, \mathrm{R}^{2}=0.315, F_{1,210}=96.65, \mathrm{p}<0.001$ ， short type: $\mathrm{n}=57, Y=0.024 X+5.745, \mathrm{R}^{2}=0.156$, $F_{1,55}=10.17, \mathrm{p}<0.01$, Fig. 2B).

Morphological observations on each spermatophore type showed that sperm mass ejaculated from short type spermatophores had a drop-like shape (Fig. 3A) and those ejaculated from long type spermatophores had a rope-like shape (Fig. 3B). Of the sperm mass attached to different female body parts, that surrounding the seminal receptacle was drop-like in shape ( $\mathrm{n}=39$ females, Fig. $3 \mathrm{C}$ ). In contrast, all of the sperm mass attached to the female oviducts was rope-like in shape $(\mathrm{n}=62$ females, Fig. 3D).

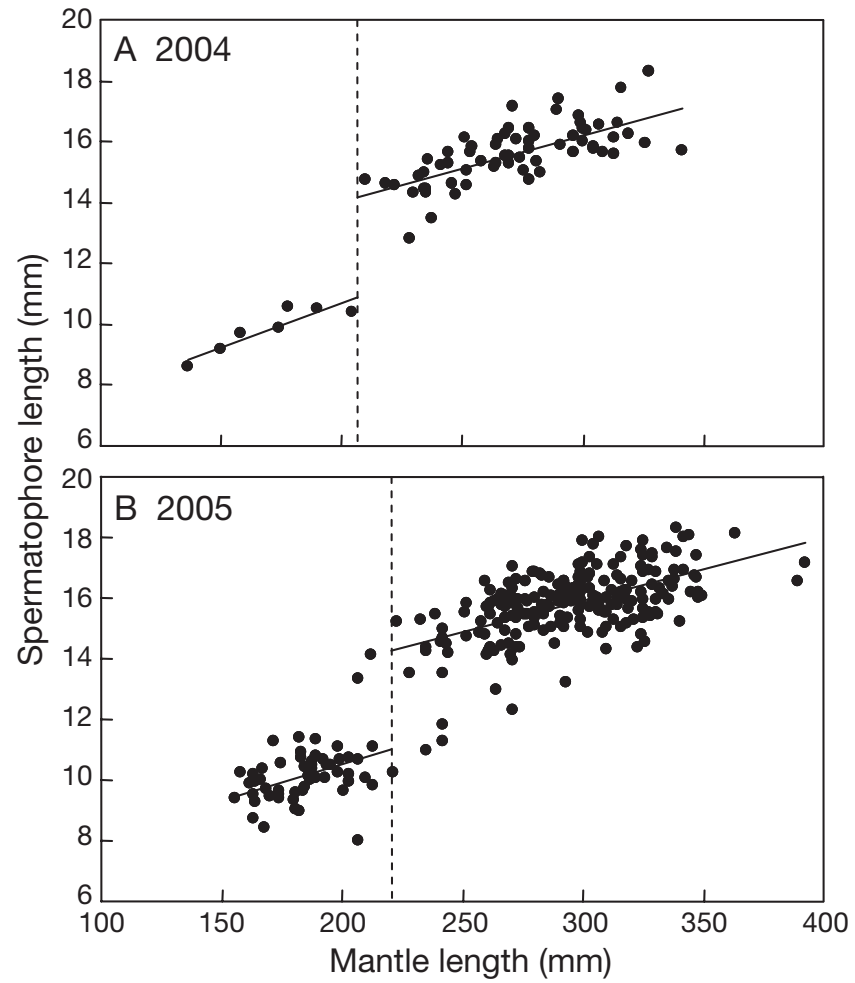

Fig. 2. Loligo bleekeri. Relationship between mantle length and spermatophore length in (A) 2004 ( $\mathrm{n}=72$ ) and (B) 2005 ( $\mathrm{n}=269$ ). Dashed vertical line shows morphological switch point: mantle length (A) $207 \mathrm{~mm}$; (B) $221 \mathrm{~mm}$

\section{DISCUSSION}

Most alternative mating tactics involve dimorphism in secondary sexual characteristics (Andersson 1994). In Loligo bleekeri, where males generally become larger than females, mature body size is a trait that 
shows secondary sexual development. In our results, mature males showed body size dimorphism in 2005, although such a clear bimodality was not observed in 2004. Because loliginid squid have an annual life span (Boyle \& Rodhouse 2005), the bimodality and the difference in size distribution between the 2 years studied is not due to age classes. The difference in size distribution between years might be related to oceanographic and biological factors such as water temperature and food availability. Hectocotylus length in 2004 and fin width in 2005 showed a nonlinear relationship with mantle length in Model 1. However, these results were not consistent for 2 years studies and no significant switch point was detected for these body parts in Model 2 and Model 3 in both years. There was also no morphological discontinuity in relation to male body size in the other 4 external characteristics (fin length, head width, mantle width and tentacle length). In contrast, spermatophore length showed clear discontinuity relative to body size in both years. Previous studies have shown that small males tend to adopt sneaking copulation behavior in the head-to-head position (Loligo pealei: Hanlon et al. 1997, L. vulgaris reynaudii: Hanlon et al. 2002). Although the behavioral-switch body size is unknown, small males with short spermatophores and large males with long spermatophores would adopt sneaking copulation in the head-to-head position and pair copulation in the male-parallel position, respectively. This suggestion is consistent with the results of our observations on each sperm mass type attached to the different female body parts.

One possible hypothesis that may explain the dimorphism of spermatophores would be quantitative and morphological adaptation of the sperm mass to the morphology of the female's sperm storage organs. Some studies have demonstrated that morphological variation in male genitalia and sperm characteristics coevolved with the morphology of the seminal receptacle in females (Pitnick et al. 2003, Hosken \& Stockley 2004). A further study to examine the morphology and the volume of the respective sperm storage sites would be required to test this hypothesis.

Another possible hypothesis relates to sperm allocation. No evidence exists to indicate that spermatophore length and sperm number are related, but short spermatophores in small males may not coincide with the prediction from the sperm competition theory which assumes a 'fair raffle principle', i.e. that sneaker males should ejaculate relatively more sperm. In loliginid squid, eggs being spawned will meet sperm stored in
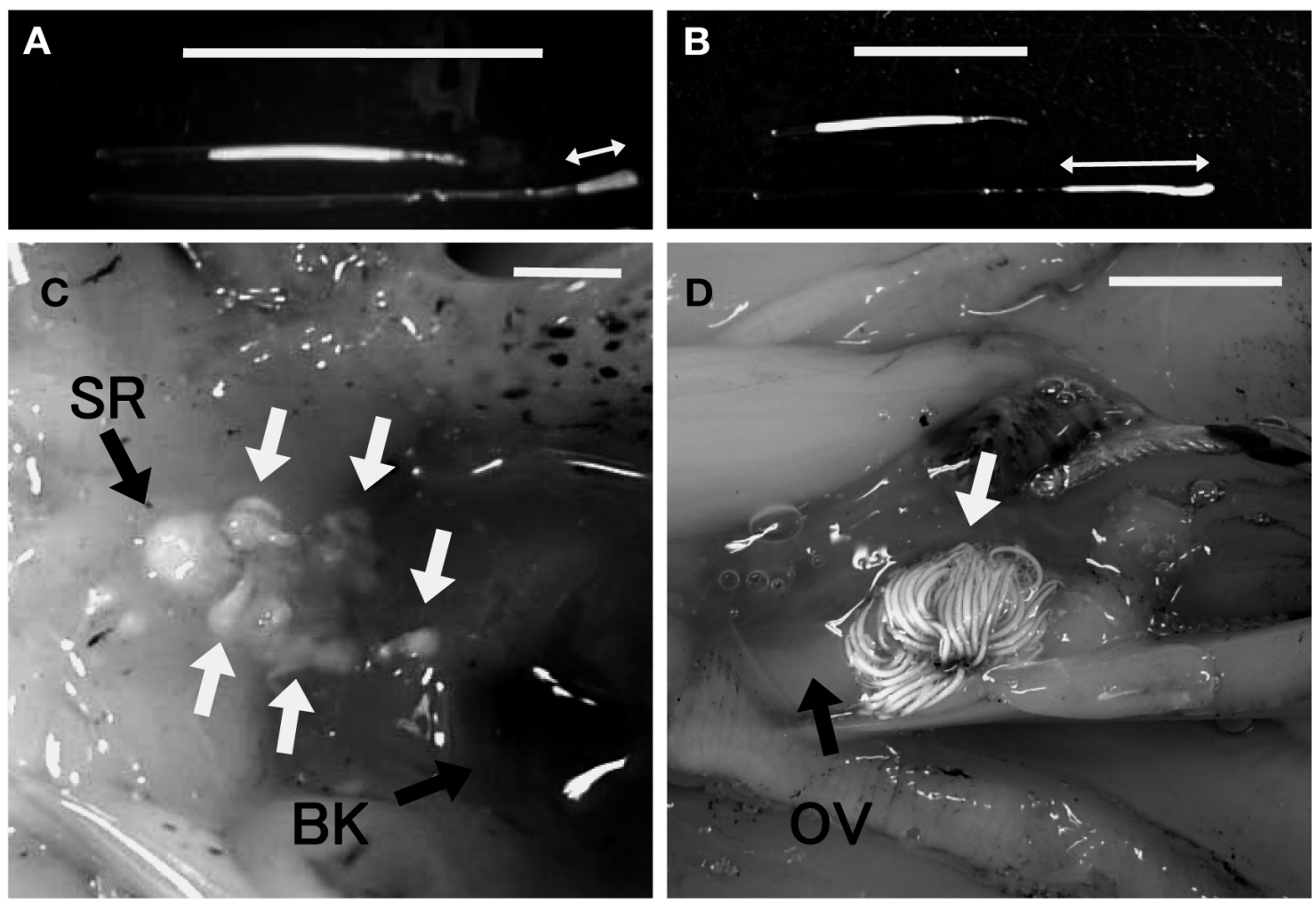

Fig. 3. Loligo bleekeri. Typical morphology of spermatophores and ejaculated sperm mass. (A) Short spermatophore (above) and a drop-like shaped ejaculated sperm mass (below, white arrow); scale bar $=10 \mathrm{~mm}$. (B) Long spermatophore (above) and a ropelike shaped ejaculated sperm mass (below, white arrow); scale bar $=10 \mathrm{~mm}$. (C) Sperm mass (white arrows) near the seminal receptacle (SR) near the beak (BK) of a female; scale bar $=5 \mathrm{~mm}$. (D) Sperm mass (white arrow) attached to the oviduct

$(\mathrm{OV})$ of a female; scale bar $=10 \mathrm{~mm}$. The oviduct was dissected to show the inside where the sperm mass was attached 
the oviduct before sperm stored in the seminal receptacle near the mouth. Fertilization success is far lower in head-to-head copulation than in paired male-parallel copulation (Iwata et al. 2005) because the number of eggs available for fertilization would be severely restricted. Several studies have shown that males adjust the number of sperm released based on the expected number of eggs available (e.g. males release more sperm when mating with larger females; Marconato \& Shapiro 1996, Sato et al. 2006). In loliginid squid, the fertilization success of head-to-head copulation events might not dramatically increase with an increase in sperm number due to a limited availability of eggs, and strategic allocations of sperm to more than a single female might be adaptive for small males. Furthermore, it has been recently suggested that males under sperm competition invest energy not only in sperm number but also in sperm quality features, such as size, velocity and mobility (Leach \& Montgomerie 2000, Vladiç \& Järvi 2001, Uglem et al. 2001). Investment in sperm number must be maintained at some minimum requirement to ensure fertility, but with limited resources available for reproduction, there is likely to be a trade-off between sperm number and quality (Snook 2005). Studies comparing sperm number and quality (size, velocity and mortality) in long and short type spermatophores would provide more insight into the adaptive value of the spermatophore dimorphism found in this study.

To our knowledge, this study is the first to report dimorphism in ejaculate characteristics in cephalopods. The morphological switch in spermatophore length would be associated with the 2 mating tactics: large males pass sperm with long spermatophores to the oviduct opening by male-parallel copulation, and small males pass sperm with short spermatophores to the seminal receptacle by head-to-head copulation. Our results suggest that alternative reproductive behaviors promote internal morphological adjustment based on the different roles that males play in the sperm competition game.

Acknowledgements. We thank Drs. J. R. Bower, Y. Watanuki and A. Takahashi for critically reading the manuscript and offering many valuable comments. We also thank Dr. K. Ito for his support in sampling. This study was partly funded by a Science Research Grant from Hakodate City, Japan.

\section{LITERATURE CITED}

Andersson M (1994) Sexual selection. Princeton University Press, Princeton, NJ

Boyle PR, Rodhouse PG (2005) Cephalopods: ecology and fisheries. Blackwell Publishing, Oxford

Eberhard WG (1982) Beetle horn dimorphism: making the best of a bad lot. Am Nat 119:420-426

Eberhard WG, Gutiérrez EE (1991) Male dimorphism in beetles and earwigs and the question of developmental constraints. Evolution 45:18-28

Evans JP, Pierotti M, Pilastro A (2003) Male mating behavior and ejaculate expenditure under sperm competition risk in the eastern mosquitofish. Behav Ecol 14:268-273

Fraley C, Raftery AE (2002) Model-based clustering, discriminant analysis, and density estuimaiton. J Am Stat Assoc 97: $611-631$

Gage AR, Barnard CJ (1996) Male crickets increase sperm number in relation to competition and female size. Behav Ecol Sociobiol 38:349-353

Gross MR (1996) Alternative reproductive strategies and tactics: diversity within sexes. Trends Ecol Evol 11:92-98

Hanlon RT, Messenger JB (1996) Cephalopod behaviour. Cambridge University Press, Cambridge

Hanlon RT, Maxwell MR, Shashar N (1997) Behavioral dynamics that would lead to multiple paternity within egg capsules of the squid Loligo pealei. Biol Bull (Woods Hole) 193:212-214

Hanlon RT, Smale MJ, Sauer WHH (2002) The mating system of the squid Loligo vulgaris reynaudii (Cephalopoda, Mollusca) off South Africa: fighting, guarding, sneaking, mating and egg laying behavior. Bull Mar Sci 71:331-345

Hosken DJ, Stockley P (2004) Sexual selection and genital evolution. Trends Ecol Evol 19:87-93

Iwata Y, Munehara H, Sakurai Y (2005) Dependence of paternity rates on alternative reproductive behaviors in the squid Loligo bleekeri. Mar Ecol Prog Ser 298:219-228

Leach B, Montgomerie R (2000) Sperm characteristics associated with different male reproductive tactics in bluegills (Lepomis macrochirus). Behav Ecol Sociobiol 49:31-37

Marconato A, Shapiro DY (1996) Sperm allocation, sperm production and fertilization rates in the bucktooth parrotfish. Anim Behav 52:971-980

Nicholls EH, Burke T, Birkhead TR (2001) Ejaculate allocation by male sand martins, Riparia riparia. Proc R Soc Lond B Biol Sci 268:1265-1270

Parker GA (1990a) Sperm competition games: raffles and roles. Proc R Soc Lond B Biol Sci 242:120-126

Parker GA (1990b) Sperm competition games: sneak and extrapair copulations. Proc R Soc Lond B Biol Sci 242:127-133

Parker GA, Ball MB, Stockley P, Gage MJG (1997) Sperm competition games: a prospective analysis of risk assessment. Proc R Soc Lond B Biol Sci 264:1793-1802

Perez JAA, Aguiar DC, Oliveira UC (2002) Biology and population dynamics of the long-finned squid Loligo plei (Cephalopoda: Loliginidae) in southern Brazilian waters. Fish Res 58:267-279

Pitnick S, Miller GT, Schneider K, Markow A (2003) Ejaculate-female coevolution in Drosophila mojavensis. Proc R Soc Lond B Biol Sci 270:1507-1512

Sato T, Ashidate M, Jindo T, Goshima S (2006) Variation of sperm allocation with male size and recovery rate of sperm numbers in spiny king crab Paralithodes brevipes. Mar Ecol Prog Ser 312:189-199

Snook RR (2005) Sperm in competition: not playing by the numbers. Trends Ecol Evol 20:46-53

Taborsky M (1998) Sperm competition in fish: 'bourgeois' males and parasitic spawning. Trends Ecol Evol 13:222-227

Uglem I, Galloway TF, Rosenqvist G, Folstad I (2001) Male dimorphism, sperm traits and immunology in the corkwing wrasse (Symphodus melops L.). Behav Ecol Sociobiol 50:511-518

Vladiç TV, Järvi T (2001) Sperm quality in the alternative reproductive tactics of Atlantic salmon: the importance of the loaded raffle mechanism. Proc R Soc Lond B Biol Sci 268:2375-2381 